\title{
PULMONARY THROMBOSIS AND ITS ASSOCIATION WITH UNEXPECTED DEATH IN CHILDHOOD
}

\author{
BY \\ JOHN L. EMERY \\ From the Department of Pathology, the Children's Hospital, Sheffield
}

(RECEIVED FOR PUBLICATION JANUARY 23, 1953)

Thrombosis of pulmonary vessels unassociated with local sepsis or cardiac deformity is rarely recognized in infancy and childhood. The condition is not usually considered by clinicians or by pathologists doing necropsies in this age period, and I have been unable to find a paper discussing this subject in the available literature.

This communication is concerned with pulmonary vascular thrombosis in 15 children seen in a series of approximately 200 consecutive necropsies.

\section{Material and Methods}

The material consisted of routine necropsies at two children's hospitals, and also cases referred through the Coroner.

A particular necropsy method was used, not for the purpose of finding vascular thrombi but in an attempt to leave fluids and inflammatory exudates in the respiratory passages in their natural position so that their distribution could be studied later in sections. In most instances the trachea was tied with a cord or clamped with artery forceps before opening the body cavities. The heart and lungs were removed together from the body, and the lungs were not handled with the fingers or compressed. The cavities of the heart were opened for general inspection. The lungs and the heart were then placed under cotton wool in fixing fluid, and the lungs cut after fixing from 36 to 48 hours. Blocks for histology were taken from the lungs as far as possible at right angles to the direction of the bronchi, and treated by routine methods.

Cases were only included when no sepsis was found in the lungs. Thrombosis was recognized on histological criteria only and cases in which the thrombi were not certainly ante-mortem in type were recorded as negative. Cases with congenital deformity of the heart were not examined for thrombi and thus these were automatically excluded from the series.

The 15 cases fell into four distinct clinical groups:
I, in the neonatal period (two cases); II, terminal incidents in children with chronic disease (four cases); III, children admitted to hospital with acute severe illness (five cases); IV, children who had been referred to the Coroner on account of their death being unexpected (four cases).

Since these groups present different problems the cases will be presented and discussed in these groups.

\section{Neonatal Period}

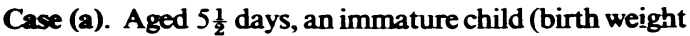
$4 \mathrm{lb}$. $14 \mathrm{oz}$.) when 24 hours old had shown signs of cerebral irritation, and at 4 days was thought to be spastic. He had no cough. In the terminal two days sclerema developed associated with a subnormal temperature. At necropsy, ante-mortem thrombosis was present in the lungs with small zones of fresh haemorrhage from alveoli. Thrombosis was also present in the larger cerebral vessels, choroid plexus and renal veins. The larger thrombi in the lung appeared histologically to be of the same age as those in the renal vessels.

Case (b). This was a premature infant aged 9 days. Cyanotic attacks began when he was aged 5 days. There was a sudden rise in temperature to $101^{\circ} \mathrm{F} .18$ hours before death. For a few hours before this the child had passed loose stools and vomited (see Fig. 1). A clinical diagnosis of respiratory failure or aspiration of vomit was made. At necropsy, certain organs were seen to be immature. Thrombi were present in cerebral vessels and the brain showed kernikterus. Thrombi were present in the lung, and there was also a small amount of oedema in the alveoli.

Both of these children were initially extremely 'weakly' but in both the immediate factor in death seemed to be generalized vascular thrombosis in which the lung vessels played a minor part in the first case, but probably the major part in the second. Thrombosis of the choroid plexuses and cerebral vessels is well known in the neonatal period (Craig, 1938). The only reported case I have found with associated pulmonary thrombosis is that recorded by Cruickshank (1930) who found thrombosis of pulmonary vessels in one case among 30 cases showing 


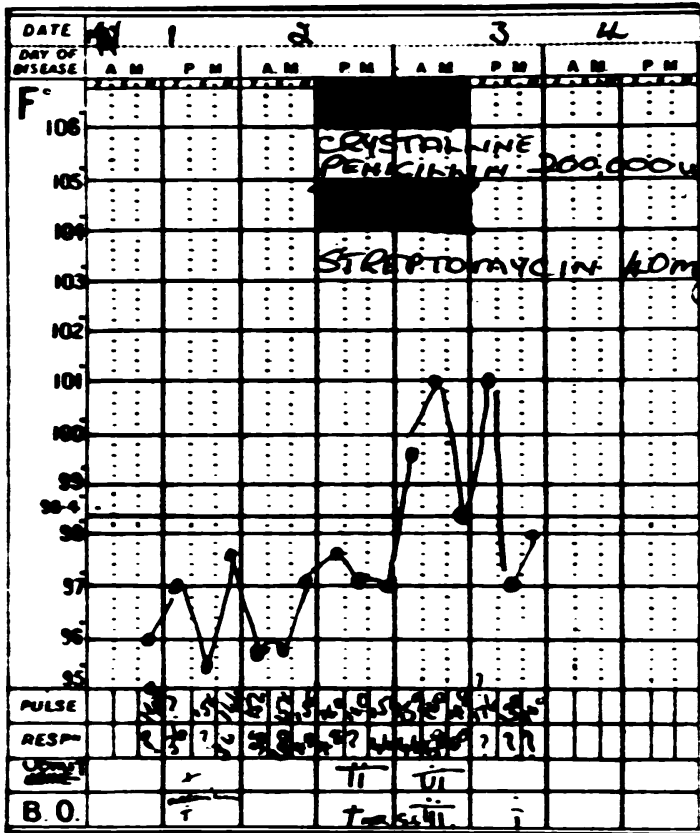

Fig. 1.- Photograph of the temperature chart of Case Ib showing pyrexia and the incidence of vomiting and diarrioea.

venous thrombosis elsewhere in a series of 800 neonatal deaths. We have no direct clue as to the cause of the thrombosis in the first case. In the second case the vomiting and frequent stools suggested an associated blood fluid disturbance. In the first case the sclerema was probably related to circulatory inadequacy and in both cases circulatory failure probably preceded the thrombosis.

\section{Terminal Incidents after Wasting and Long-standing Sepsis}

Case (a). Aged 10 months, this child had a gangrenous intussusception at the age of 8 months. Wound sepsis followed with a fistula and chronic sepsis. The child continued in a debilitated state for two months. Three days before death there was a rise in temperature and respiration rate, and cyanosis (Fig. 2). This persisted with no response to penicillin and streptomycin. The clinical diagnosis was pneumonia. Before the pyrexia the child had passed loose stools and a greater number of loose stools were passed on the first day of pyrexia. At necropsy, chronic peritonitis was present with a few loculations of pus. There was recent thrombosis of the inferior vena cava and pelvic veins. In the lung there was thrombosis of many vessels and a small amount of oedema.

Case (b). A boy aged 3 years had been ill for three months with persistent vomiting and considerable wasting. There was necrosis of the gums and finger nails and also desquamation of the skin, particularly of the hands and feet. The clinical diagnosis was acrodynia.
Two hours before death there was a sudden onset of dyspnoea with moist sounds in the chest. The temperature was not raised. At necropsy, there was thrombosis in the inferior vena cava and also in some hepatic veins. In the lung there was extensive thrombosis throughout all the major vessels, both arteries and veins, and some alveolar oedema. The thrombi in the lung did not appear to be more recent than those in the inferior vena cava or hepatic veins.

Case (c). Aged 5 months, this child had shown mental retardation from birth. At the age of 3 months he presented with increasing jaundice and a laparotomy was performed. He was finally admitted with a history that pus had been discharging for two days from the abdominal wound. Two days before death the temperature suddenly rose. This was associated with the bowels being opened three times a day (Fig. 3). The clinical diagnosis was septicaemia. At necropsy, the underlying disease was chronic hepatitis with extensive liver damage and fibrosis. In the lung many vessels were thrombosed and there was some alveolar oedema.

Case (d). This child aged 8 weeks was prematurely born of a mother with syphilis. He was admitted to hospital with a generalized rash of specific pemphigus and with rhagades, sore buttocks and a history of snuffles and nasal discharge from birth. There had been recent epistaxes. Although ill, the child's condition did not cause acute concern. He unexpectedly collapsed and died within 15 minutes of the onset of stertorous breathing. At necropsy, multiple miliary gummata of the liver were present. Spirochaetes were demonstrated in the liver and adrenal glands. There were recent antemortem thrombi in vessels of the kidneys and in the main

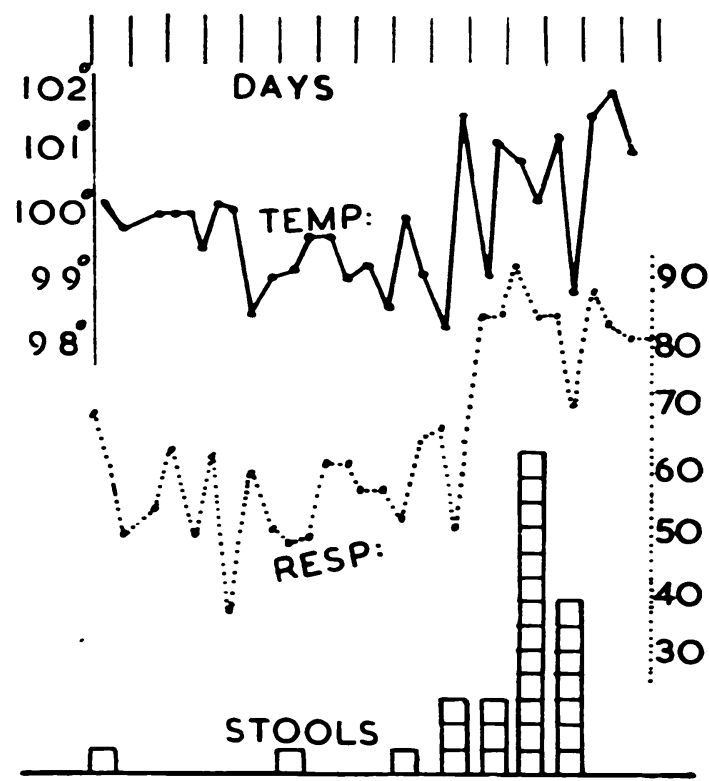

Fig. 2.-Diagram of the temperature and respiration rate of Case IIa showing also the number of stools passed. 


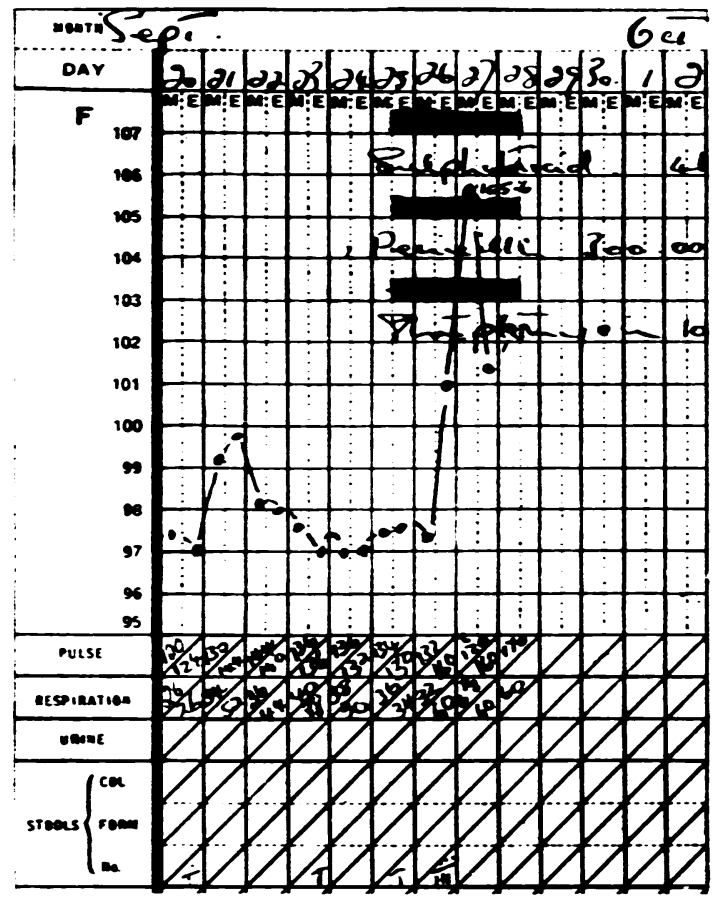

Fig. 3.-Photograph of the temperature chart in Case IIc.

pulmonary veins. There was a small amount of alveolar oedema. No evidence of inhalation was found.

These children were all severely ill with different diseases and venous thrombosis appeared to be the precipitating terminal incident. In two cases (b) and (d) death was very rapid with symptoms similar to those of inhalation of regurgitated stomach contents or acute left heart failure. The other two cases presented a definite clinical picture with a raised temperature and respiratory rate showing no response to chemotherapy or antibiotics.

A rise in temperature was described by Bailey and Hass (1937) in association with cerebral sinus thrombosis in infants. This rise in temperature was well recognized in the older literature in adults. Möller (1922) in an extensive review of pulmonary thrombosis in adults describes the 'Michaelis' symptom which consists of a marked rise in temperature for no apparent reason-a symptom indicating hidden venous thrombosis.

The rise in temperature and onset of symptoms in cases (a) and (c) seem to be directly related to an increase in the number of stools, reflecting an increase in fluid output. In each case the change in stools preceded the pyrexia by only a few hours. While we have no measurements of blood viscosity in these cases the concentration of the erythrocytes in the blood vessels throughout the body suggested a diminution in the fluid compartment of the blood. This was not seen in the other two cases and we have no clue as to the cause of the thrombosis. The only case I have seen recorded was by Ribadeau-Dumas and Chabrun (1927) who describe the necropsy finding of pulmonary venous thrombosis in a 5month-old child ill with pneumococcal meningitis; in their case lung infection was also present.

\section{Cases Admitted to Hospital with Acute Severe Illness}

Case (a). A premature infant, aged 10 months on admission, had retrolental fibro-dysplasia. The child had been 'unwell' for two and a half days. Two to three hours before death he began vomiting with convulsions. The child died almost as soon as he reached hospital and no temperature was recorded. At necropsy, there was thrombosis of the longitudinal sinus, cerebral vessels and parts of the choroid plexus, and in large vessels of the lung. There was some oedema in the alveoli. The connective tissue of the body suggested general dehydration.

Case (b). A baby aged 8 months had a history of a 'cold' for three weeks. The child was admitted with a history of convulsions for one day. On examination there were crepitations in the chest and meningismus. The child died almost immediately upon admission. At necropsy no basic disease was found other than upper respiratory infection. There was thrombosis of cerebral sinuses, part of the choroid plexus and many vessels in the lung.

Case (c). A girl aged 16 months had had an attack of what was diagnosed as 'asthma' two months previously. and was admitted with acute respiratory symptoms suggesting asthma, but no respiratory wheeze was heard on auscultation. The temperature rose to $104^{\prime} \mathrm{F}$. The child died within $\mathbf{4 8}$ hours of the acute symptoms. The clinical diagnosis was acute laryngo-tracheo-bronchitis. There had been no diarrhoea or vomiting. At necropsy, no gross lesions were found outside the lung. The lung. in addition to thrombosis, showed acute laryngo-tracheobronchitis of the exudative type and vascular thrombosis. There was some oedema in the alveoli. No thrombi were found in other organs.

Case (d). Aged 3 months, this child had had no previous illness. He had been 'off' his food for nine days. On the day before admission grunting respirations began with pulse 164 and temperature $100^{-} 5^{\prime} \mathrm{F}$. On admission the child had a distended, rigid abdomen and died while under operation within 48 hours of the onset of the symptoms. At necropsy, there was acute generalized peritonitis from rupture of a retro-umbilical abscess which had probably arisen in a urachal cyst. Thrombosis was present in cerebral vessels as well as in the lung. There was a small amount of oedema in the lung.

Case (e). A child aged 7 months had had no previous illnesses, but had had a slight cough for three days, had vomited occasionally for two days, and had loose stools on the day before admission. Severe symptoms lasted 12 hours only, and consisted of laboured breathing, temperature $103^{\circ} \mathrm{F}$. and a great increase in pulse rate. The child 
was admitted as a case of acute bronchopneumonia and died almost immediately. At necropsy, no underlying disease was found other than mild acute upper respiratory infection. There was extensive thrombosis throughout most of the veins of the lung.

In two of these cases ( $a$ and $b$ ) the clinical picture was complicated by thrombosis occurring in the cerebral as well as in the pulmonary vessels. In one case (d) the pulmonary thrombosis was immediately related to acute peritonitis. In this case, without careful study, the death would have been ascribed to 'operative shock'. In the other two cases the pulmonary thrombosis occurred alone, and was apparently a major factor in death. The necropsy diagnosis without a complete histological study would have been acute bronchopneumonia.

In these cases the history of vomiting or diarrhoea seen in the previous group is also present. Whether a more complete history would reveal further symptoms it is not possible to say, but the experience that has been gained by 'triple' histories taken in coroner's cases suggests that the history obtained in acute diseases is often quite misleading.

\section{Sudlea Deaths Referred to the Coroner}

Case (a). An immature infant (birth weight $4 \mathrm{lb} .2 \mathrm{oz}$ ) aged 3 weeks was referred to the Coroner as a sudden death. Later a history of loose stools for one day was obtained. At necropsy, the child appeared to be dehydrated. Thrombosis of both pulmonary and cerebral vessels was present, also a small amount of oedema of the lung.

Case (b). The child, aged 4 weeks, was found dead in his cot. He had had a 'cold' for three days and had been off his food for three days. At necropsy, there was massive thrombosis of pulmonary veins and also of small veins of the kidney, and some oedema of the lung.

Case (c). A child aged 7 weeks, was found dead in his cot. He had had a 'sticky' eye for one week before death. At necropsy, no underlying disease was found. Thrombosis of pulmonary, renal and cerebral veins was present.

Case (d). An infant aged 6 months was found dead in his cot. There was a history of diarrhoea for one day. The child was reputed to be teething. At necropsy, the only abnormality found was thrombosis in pulmonary vessels and a small amount of oedema of the lung.

Unexpected deaths in children form one of the most disconcerting problems in paediatric pathology. Since the decline in popularity of 'status thymaticus' the most popular finding has been that of microscopic evidence of upper respiratory infection. Such microscopic inflammatory foci were present in the cases listed above, but in no greater degree than is seen in other children with otherwise adequate causes of death. The history of digestive upset in three of the cases was not obtained at the time of the necropsy but several days later by the hospital almoner visiting the children's homes when the alarm of the sudden death and inquest had subsided.

\section{Discussion}

Thrombosis of renal vessels associated with diarrhoea in infancy has been recognized since 1837 (Raynor), and by 1877 (Hutinel) 42 cases had been reported. Thrombosis of the choroid plexus (Craig, 1938) and of the cerebral sinuses, designated 'marantic thrombosis' by Virchow, is similarly well known, but thrombosis of the vessels of the lung in childhood seems to have been overlooked. The cause of this is probably technical as cerebral sinuses and renal veins are obvious at necropsy and changes in colour of the brain and kidney call for elucidation. The pulmonary vessels on the other hand are not so visible, and congestion and consolidation of the lung is so linked with the idea of pneumonia that detailed study of the content of the pulmonary vessels is generally overlooked. Until the post-mortem technique of routine fixing of the lungs with the heart was instituted we did not see pulmonary thrombi. Most lung sections give a false idea of the vascularity of the normal lung. It is surprising how few of the larger vessels contain blood in routine sections of the lung.

Belt (1934) points out that pulmonary thrombosis is found in about $10 \%$ of adult medical necropsies and is frequently not associated with embolism. Spain and Moses (1946) found pulmonary thrombosis in 109 of 1,000 necropsies in persons over the age of 20. Brenner (1935) in an extensive study of pulmonary vascular disease, remarks 'It is clear that thromboses are commonly present at autopsy but that only careful search will reveal them in many cases'.

Thrombosis is also known to occur in adults in the presence of lung infection and diseases such as silicosis (Geever, 1947) and tuberculosis (Jones, Black and Sparr, 1950) and may itself produce the terminal incident in these diseases. The child reported by Ribadeau-Dumas and Chabrun (1927) would seem to belong to this group. Eppinger and Wagner (1920) describe pulmonary venous thrombosis in adults associated with primary pulmonary sclerosis. They illustrate a small vessel with extensive intimal proliferation such as was seen in a single vessel in one case in the present series.

The rise in temperature seen in many of the cases in groups I and II seems to be associated with vascular thrombosis irrespective of the site or cause of the thrombosis. In infants it has been observed with cerebral sinus thrombosis (Bailey and Hass, 


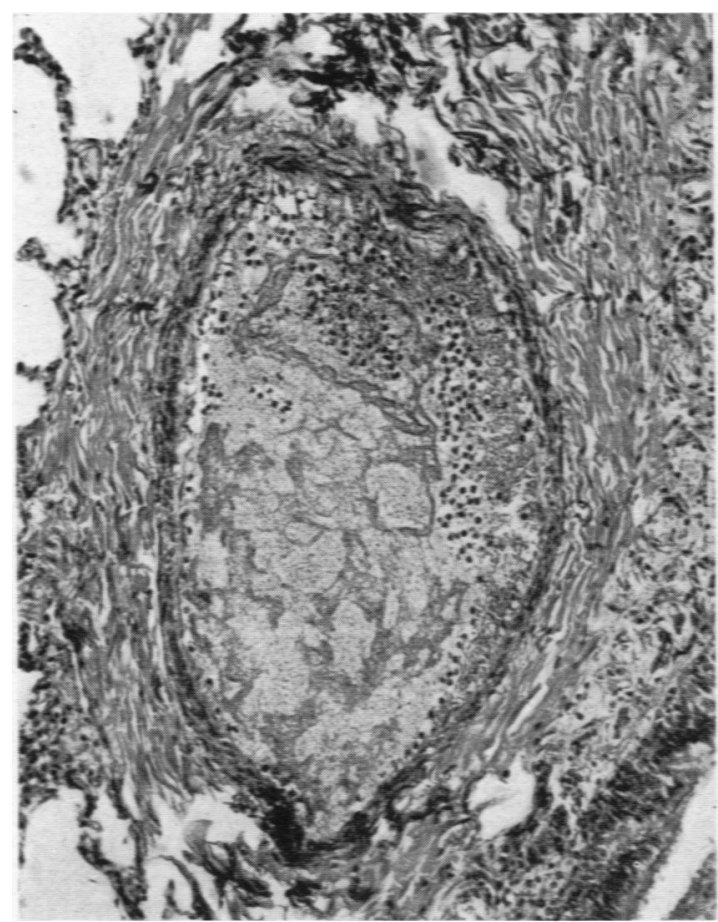

Fig. 4.-Ante-mortem thrombus in a small vein. Masson trichrome $\times 110$.

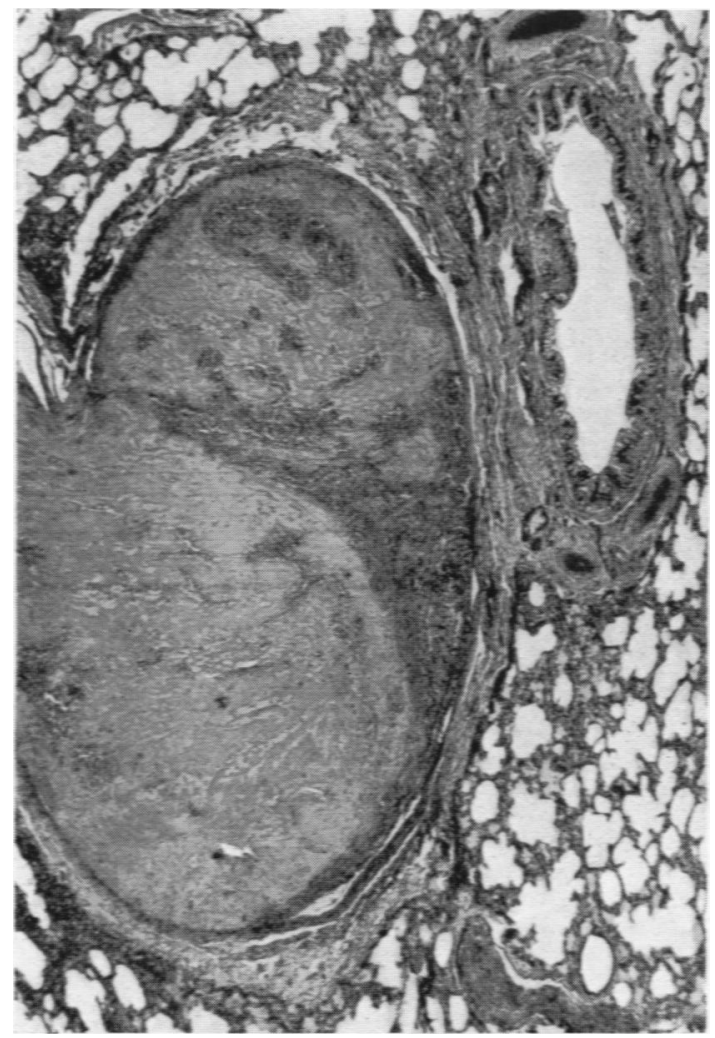

Fic. 5.-Thrombus in a large vein near a small bronchus. Masson trichrome $\times 40$.

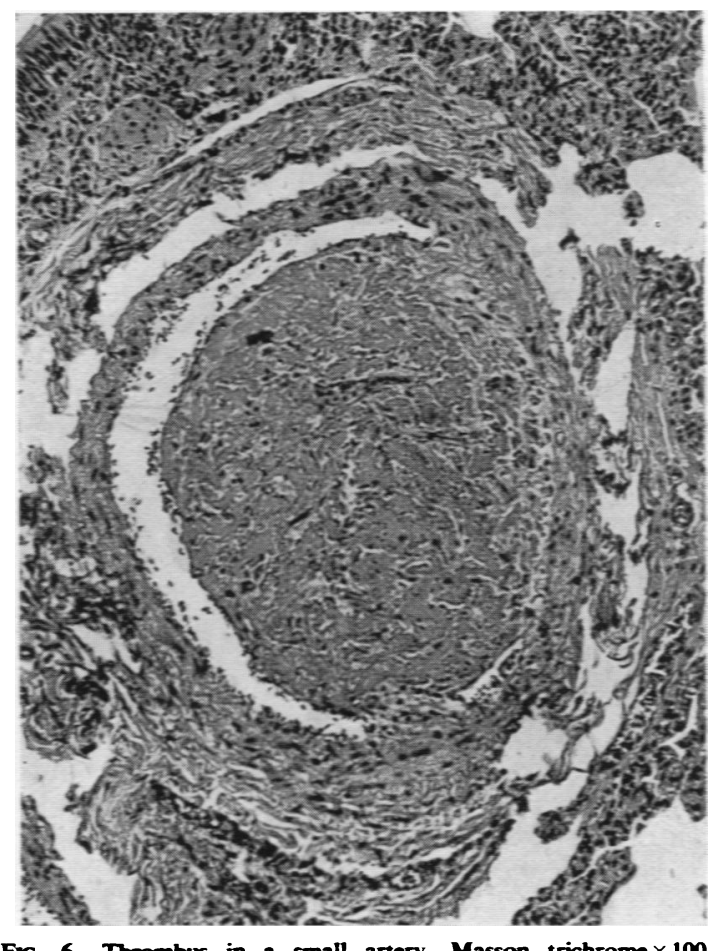

Fig. 6.-Thrombus in a small artery. Masson trichrome $\times 100$.

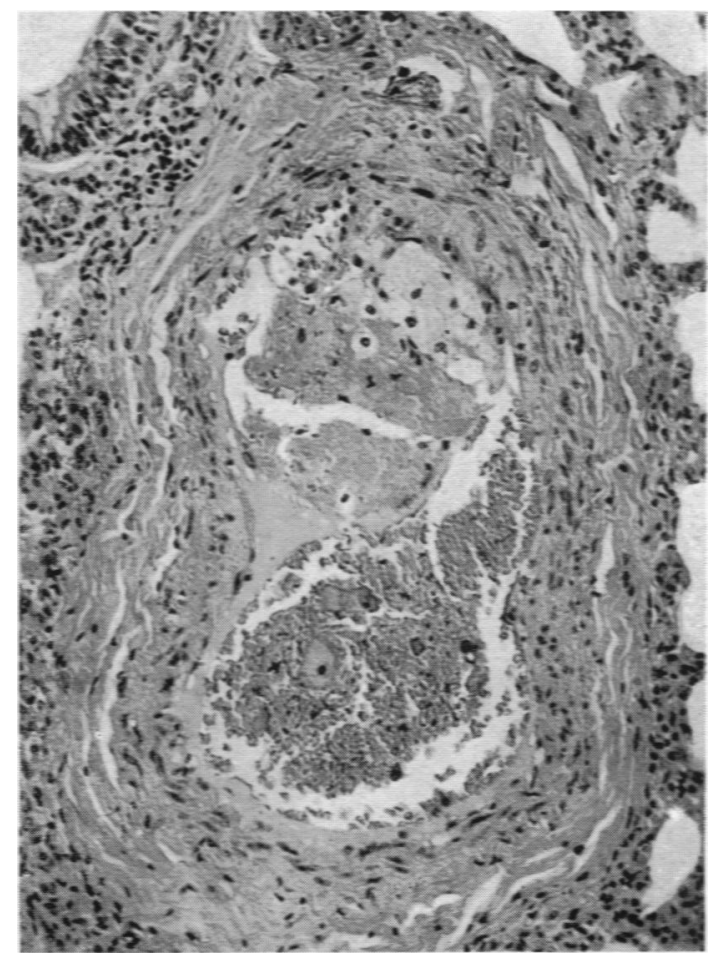

Fic. 7.-A thrombus partially occluding a small vessed and 'foamy' cell proliferation of the underlying intima. Masson trichrome $\times 150$.

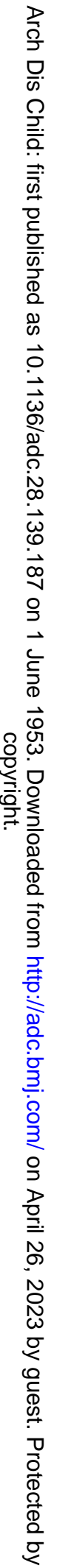


1937) and in adults has long been recognized in pulmonary thrombosis as the 'Michaelis symptom' (Möller, 1922). The importance of this symptom is probably increasing. Kallner (1948) considers that thrombosis of vessels in the lung in pneumonia is commonly why the temperature does not fall during antibiotic therapy. He noted a clinical response in these cases to anticoagulant therapy.

The absence of infarction in the present cases is not surprising. The experimental work of Chapman, Gugle and Wheeler (1949) suggests that embolism does not produce infarction of the lung unless some other pulmonary or circulatory factors are present. The small amount of alveolar haemorrhage seen in many cases may well represent minute infarction but such small haemorrhages are almost universal in deaths in infants. Hepler (1934), discussing thrombosis of renal veins, attempts to classify the thromboses on the site of the original thrombosis within the kidney but this seems to be of academic interest only, and I was unable to make any conclusive observations in the lungs. Vessels of all sizes seem to be affected in different cases but never all in the same case (Figs. 4, 5 and 6).

Abeshouse (1945) reviewed 228 cases of renal venous thrombosis from the literature and of these 98 occurred in the first year of life. Morison (1945) in one of his cases of renal thrombosis found thrombi in small pulmonary arteries-which he considers to be due to emboli of the kidney. It would seem from our cases that the same factors as those producing renal and cerebral thrombosis also cause pulmonary thrombi. Sandblom (1948) discussing renal thrombosis suggested that two types of thrombosis occurred in childhood, a rare, primary type occurring during the first week of life and a secondary, commoner type associated with dehydration. It would be simple to suggest that in the cases where we found a history of diarrhoea or vomiting that thrombosis was due to secondary haemoconcentration, and in the other cases that the condition was primary. But such an explanation would be a little naive. Many children with very severe dehydration do not have thromboses, and factors such as endothelial cells, acute circulatory failure, changes in factors related to blood coagulability such as antithrombin, may each play a precipitant role in different cases.

Zuelzer, Kurnetz and Fallon (1951) in describing thrombosis in infancy consider the chief to be haemoconcentration, slowing of the blood stream and damage to the endothelium. The only vascular endothelial change seen in the present cases were one (Fig. 7) showing intimal changes probably secondary to thrombosis and the other an endarter- itis of long standing in a single vessel. Without more evidence it seems pointless to speculate further as to the aetiology of the thrombosis; the most fruitful cases for study seem to be those in Group II where vascular thrombosis occurs while the patients are in hospital under treatment.

It is not justifiable to say that pulmonary thrombosis was the primary cause of death in these children. The presence of ante-mortem thrombi and of alveolar oedema does suggest that the lesion was the terminal încident. Those children having symptoms before death suggest that the pulmonary thrombosis was a major factor in death. In the children who were observed in hospital before the onset of pulmonary thrombosis an increased loss of fluid from the body had occurred. This suggests that haemoconcentration and increased viscosity of the blood may have been a factor in the formation of the thrombosis, but we have no data on the blood coagulation in these infants.

From a clinical viewpoint, however, if an ill child develops unexpected pyrexia and dyspnoea associated with loose stools or vomiting, venous thrombosis should be actively considered, and anticoagulant therapy is indicated, particularly if there is no response to antibiotics.

\section{Summary}

Fifteen cases of pulmonary vascular thrombosis with alveolar oedema in children are described.

The cases occurred in four clinical groups: (a) severely debilitated neonates; $(b)$ children severely ill for a long period; (c) children admitted to hospital with severe dyspnoea; $(d)$ children found dead and referred to the Coroner.

In children observed before death, symptoms of pulmonary thrombosis, which consist of pyrexia and dyspnoea showing no response to antibiotic therapy, are frequently preceded by a history of loose stools or vomiting.

Care in maintaining fluid intake and tissue hydration in debilitated infants may prevent the onset of thrombosis, but we have no knowledge of the cause of the thrombosis in many cases.

Where thrombosis has occurred anticoagulation therapy would seem reasonable in addition to the usual antibiotics.

The cases in this study were under the care of Dr. T. Colver, Dr. R. R. Gordon, Dr. M. Middleton, Professor R. S. Illingworth, and Mr. R. B. Zachary, and their case notes have been freely used. The sudden deaths were referred by Mr. C. G. Lockwood. I am grateful to Miss C. Crawley for her help in obtaining case histories in the cases of sudden death.

Photographs by Mr. A. Tunstill. 
Reperences

Abeshouse. B. S. (1945). Urol. cutan. Rev., 49, 661 .

Baiky, O. T. and Hass, G. M. (1937). J. Pediat., 11, 755.

Belt, T. H. (1934). Amer. J. Path., 10, 129.

Brenner. O. (1935). Areh intern Med 561189

Chapman, D. W., Gugle, L. J. and Wheeler, P. W. (1949). Ibid., 83, 158.

Craig. W. S. (1938). Archives of Disease in Childhood, 13, 89

Cruickshank. J. N. (1930). Spec. Rep. Ser. med. Res. Coun. Land. No. 145 , p. 63

Eppinger, H. and Wasner, R. (1920). Wien. Arch. inn. Med., 1, 83

Geever, E. F. (1947). Amer. J. med. Sci., 214, 292.

Hepler, A. B. (1934). J. Urol., Belrimore, 31, 527.

Hutinel, V. (1877a). Qooted by Barenberg, L. H., Greenstein, N. M., Levy, W. and Rosenbluth, S. B. (1941). Amer. J. Dis. Child.,
- (1877b) 'Contributions a l'étude des troubles de la circulation veineuse chez l'enfant et en particulier chez $k$ nouveau-ne.' Thesis, Paris. Delahage, Paris.

Jones, R. S., Black. T. C. and Sparr, H. A. (1950). Amer. Rev. Tuberc., 61, 826.

Kallner, S. (1948). Arch. intern. Med., 81, 126.

Möller, P. (1922). Beitr. path. Anat., 71, 27.

Morison, J. E. (1945). Archives of Disease in Childhood, 20, 129.

Raynor, P. (1837). Traités des maladies des Reins. Paris.

Ribadeau-Dumas, L. and Chabrun, L. (1927). Bull. Soc. méd. Hôp., Paris, 51, 402.

Sandblom, P. (1948). Acta Paediat., Stockh., 35, 160.

Spain, D. M. and Moses, J. B. (1946). Amer. J. med. Sci., 212, 707.

Zueber, W. W., Kurnetz, R. and Fallon, R. (1951). Amer. J. Dis. Child. 81, 27. 